\title{
Encore Romanos le mélode.
}

Les études que, de divers côtés, on a consacrées en ces derniers temps au grand poète byzantin sont loin d'avoir dissipé l'incertitude qui enveloppe depuis toujours la question chronologique, et en ce moment même, l'opinion faisant de Romanos un contemporain de l'empereur Anastase I (491-518) ne compte pas moins d'adhérents que celle qui se prononce pour Anastase II (713-716). Il y a trois ans, le triomphe des partisans du second Anastase parut assuré, lorsque M. Gelzer eut attiré l'attention sur un cantique du mélode, où se lisent

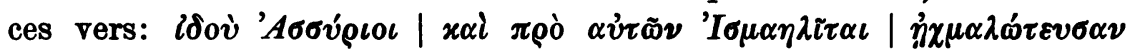
$\dot{\eta} \mu \tilde{\alpha}$ s. On sait que M. Gelzer, suivi par M. Krumbacher, traduisait ce passage de la façon suivante: «Voici que les califes abbassides de Bagdad et, avant eux, les califes ommiades de Damas nous ont amenés prisonniers. $\aleph^{1}$ ) Toutefois, cette interprétation était à peine proposée que M. de Boor lui en substituait une seconde ${ }^{2}$ ), d'après laquelle les

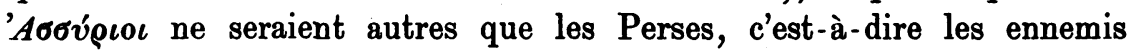
les plus acharnés de Byzance pendant la première moitié du $\mathrm{VI}^{\circ}$ siècle;

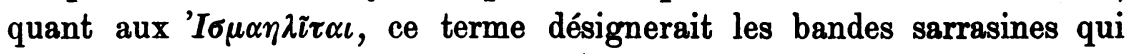
dévastèrent la Syrie, la Palestine et la Phénicie sous le règne d'Anastase I, à l'époque où la paix subsistait encore entre les Perses et l'empire grec. Le travail de M. de Boor eut pour résultat de ramener plus ou moins M. Krumbacher ${ }^{3}$ ) à l'opinion qu'il avait longuement défendue en 1897 dans son Histoire de la littérature byzantine, à savoir que Romanos est contemporain d'Anastase I.4)

1) Krambacher, Umarbeitungen bei Romanos, 1899, p. 144-145.

2) Die Lebenszeit des Dichters Romanos, Byzant. Zeitschr., t. IX (1900), p. $633-640$.

3) Romanos und Kyriakos, Sitzungsb. der philos.-philol. und der hist. Cl. der kgl. bayer. Akad. der Wiss., 1901, Heft V, p. 723.

4) On trouvera un exposé complet de la controverse, depuis ses origines, dans un récent travail de M. Vasiljev, La chronologie de Romanos le mélode (en russe), Vizantiskij Vremennik, t. VIII (1901), p. 435-478. Sans trancher définitivement la question, l'auteur apporte de nouveaux arguments en faveur d'Anastase I, 
Évidemment, la discussion ne pouvait en rester là. Le P. Vailhé vient de reprendre certains côtés du problème ${ }^{1}$ ) et il espère, grâce aux preuves nouvelles qu'il apporte, faire lâcher prise aux chæmpions du premier Anastase. Bien que l'étude de l'érudit Assomptionniste soit ingénieusement conduite, nous ferons voir que ses démonstrations n'ont guère chance d'être acceptées. Nous montrerons en même temps que si l'on doit réduire de beaucoup la valeur d'un argument invoqué tour à tour par les partisans du VIe siècle et par ceux du VIII', il est permis de le compter néanmoins parmi les indices favorables au $\mathrm{VI}^{\mathrm{e}}$ siècle. ${ }^{2}$ )

Le P. Vailhé est d'accord avec M. de Boor pour repousser la version que proposait M. Gelzer du passage du cantique de Romanos relatif aux Assyriens et aux Ismaélites, et qui plus est, il apporte sur ce point de si bonnes raisons, qu'il sera peut-être difficile de défendre encore, avec quelque espoir de succès, l'opinion du professeur de Jéna. Par contre, en ce qui concerne l'interprétation du même texte donnée par M. de Boor, le P. Vailhé «ne croit pas que l'argumentation de celui-ci soit le moins du monde concluante. Et cela pour deux motifs: d'abord, parce qu'il ne cite aucun témoignage d'écrivain byzantin qui appelle Assyriens les Perses du VI॰ siècle; en second lieu, parce qu'il n'a pas démontré que ce terme d'Assyriens n'ait pas été appliqué aux Arabes du VIII' siècle» (p. 209). Le P. Vailhé se charge précisément de fournir la preuve qu'on a appelé de ce nom les Arabes de cette époque. Dans l'une des cuvres d'un contemporain d'Anastase II, le panégyrique de saint Jacques par saint André de Crète $^{3}$ ), il a remarqué la phrase que

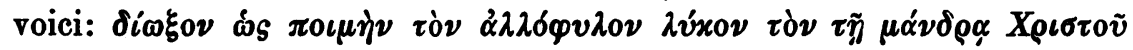

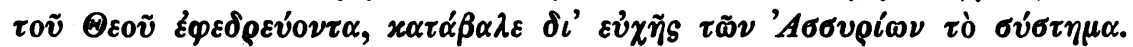

reprend et développe les preuves présentées antérieurement et s'efforce de résoudre les objections. Cette étude n'a pu être utilisée par le P. Vailhé, dont l'article a paru presque en même temps.

1) Saint Romain le mélode, Échos d'Orient, t. V (1902), p. 207-212.

2) Notre travail était déjà entre les mains du directeur de la Byz. Zeitschr., lorsqu'a paru en feuilleton dans la $N \varepsilon \alpha_{\alpha}$ ' $H \mu \varepsilon \rho \alpha$ du 22 et du 24 juillet 1902 un

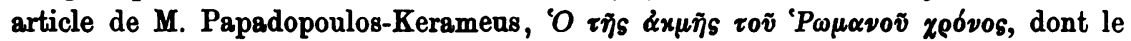
but principal est également de faire voir la faiblesse des arguments du P. Vailhé. Ce but est pleinement atteint et bon nombre des observations que nous émettons ici ainsi que le passage de la Vie de $\mathrm{S}$. Syméon Stylite le jeune publié ci-après se trouvent réunis déjà dans la note de la $N \varepsilon_{\alpha}{ }^{\circ} H \mu \varepsilon \varepsilon \alpha$. Nous croyons que notre étude conservera cependant quelque utilité, en raison du peu de publicité de ce journal en dehors des pays de langue grecque.

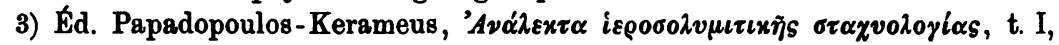
1891, p. 14. 
Or, nous dit-il, ce passage désigne évidemment les Arabes ${ }^{1}$ ), puisque, à l'époque d'André de Crète, l'empire avait pour seuls ennemis les Arabes et les Bulgares. Et «si saint André de Crète appelait les Arabes des Assyriens au commencement du $\mathrm{VIII}^{\mathrm{e}}$ " siècle, pourquoi saint Romain n'aurait-il pu le faire à la même époque?... Par les Assyriens du cantique, nous entendrons les califes arabes ou le pouvoir arabe constitué, qui, durant la première moitié du VIII ${ }^{\circ}$ siècle, infligea de nombreuses défaites aux empereurs byzantins, et par les Ismaélites, les tribus nomades, de Palestine et de Syrie, qui pillèrent ces provinces, brûlèrent les couvents et les églises, tuèrent ou firent prisonniers les moines et les chrétiens durant tout le VIII ${ }^{e}$ siècle» (p. 210). ${ }^{2}$ )

A parler franchement, cette argumentation nous parait loin d'être décisive. $\mathrm{Si}$, comme le constate le $\mathrm{P}$. Vailhé, la démonstration de M. Gelzer pour identifier les Assyriens avec les Abbassides de Bagdad n'est pas très rigoureuse, il faut avouer que la sienne mérite un peu le même reproche. Rien ne prouve, en effet, que dans la phrase d'André de Crète il soit réellement question des Arabes et même, lorsqu'on y regarde de près, cette proposition perd beaucoup de sa vraisemblance. Non seulement le célèbre homéliste ne désigne pas explicitement les Arabes sous le nom d'Assyriens, mais en outre, on conviendra, après lecture du contexte, que le passage dont fait état le $P$. Vailhé est susceptible d'une seconde interprétation aussi probable, pour le moins, que la sienne. Ce passage appartient à la prière qui termine le discours de saint André. Fait important à noter, tout ce qui précède cette invocation est exclusivement consacré au récit très objectif de la vie et de l'apostolat de saint Jacques; il n'y est jamais question, même sous forme d'allusion ou d'image, des événements contemporains de l'orateur. Pour qu'on puisse rendre sa signification exacte à la conclusion du panégyrique, il est nécessaire de citer celle-ci

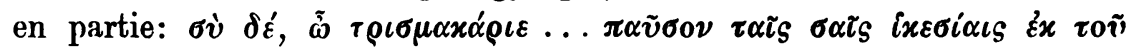

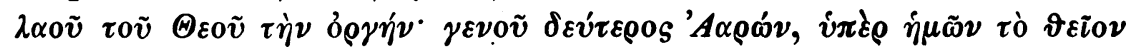

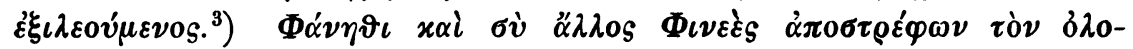

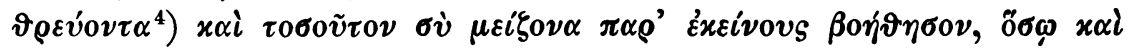

1) La même interprétation avait été proposée auparavant, sans preuve à l'appui, par M. J. Haussleiter, Zeitschrift für Kirchengeschichte, t. XIV (1893), p. 76.

2) Cette solution, qui tient le milieu entre celles de MM. de Boor et Gelzer, a sur celle de M. Gelzer l'avantage de concilier parfaitement la chronologie de la vie de Romanos avec le texte du cantique, en ne prolongeant pas l'existence du poète jusque sous la dynastie abbasside.

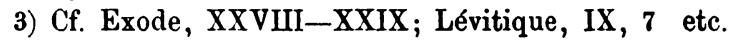

4) Nombres, $\mathrm{XXV}, 7,11$. 


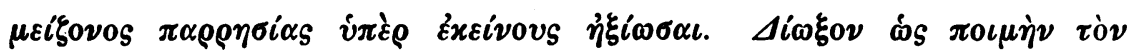

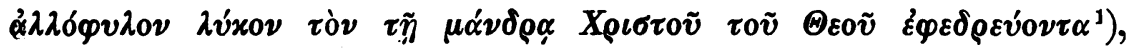

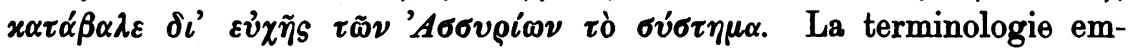
ployée ici par André de Crète étant tout entière empruntée aux Livres saints, pourquoi la phrase concernant les Assyriens ferait-elle exception? C'est ce que paraît s'être demandé le P. Vailhé lui-même, puisqu'il écrit en note: «On dira peut-être que saint André de Crète emploie une figure biblique pour désigner Léon l'Isaurien et sa bande d'iconoclastes, qui se conduisaient envers les chrétiens comme les Assyriens envers le peuple juif. Outre que cette interprétation est bien compliquée, saint André de Crète la réfute lui-même dans ce passage, lorsque, après avoir dépeint la dévastation des Églises, il demande à Dieu de fortifier l'empire, c'est-à-dire son détenteur, $\tau \grave{\eta} v$

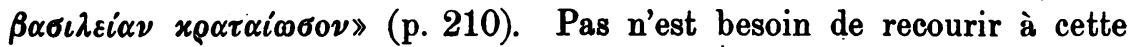
«interprétation compliquée». Saint André ne parle pas plus des Iconoclastes que des Arabes. La figure biblique dont il se sert désigne, d'une manière générale, les ennemis du peuple chrétien ${ }^{8}$ ), et sans doute comprenait-il par là les ennemis extérieurs comme ceux de l'intérieur. S'il n'était téméraire de vouloir préciser le sens de cette péroraison, aux termes trop vagues pour d'autres que les auditeurs de l'époque, on pourrait croire néanmoins que le panégyriste a visé tout particulièrement les Iconoclastes, lui qui s'écrie en terminant: «Tu vois, ô très saint, la dispersion, le ravage, la persécution, la dévastation, la profanation des églises et des autels, tous ces maux qui ont fondu sur nous à cause de nos péchés. Purifie donc l'Église qui $a$ été souillée ( pire ...». Dans cette dernière expression, le $\mathrm{P}$. Vailhé voit la preuve qu'il ne peut être question ici des Iconoclastes et de Léon l'Isaurien. Une pareille conclusion ne s'impose pas, et nous croyons que saint André était parfaitement en droit de prier pour l'affermissement de l'empire, tout en souhaitant la chute du souverain régnant. ${ }^{3}$ )

1) Jean, X, 11-12; Actes, XX, 29.

2) Les écrivains byzantins comparent parfois aux Assyriens de la Bible les nations hostiles à l'empire et à la foi. Voir, par exemple, la Vie de S. Jean Damascène, Patrol.gr., t. 94, col. 437; le Typicon de l'église de Jérusalem publié

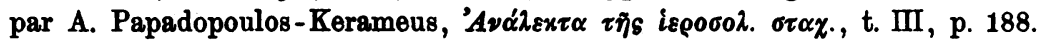

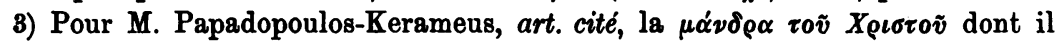
est question à la fin du discours d'Andiré de Crète, désignerait l'ensemble des chrétiens de l'empire grec, ou plutôt l'église de Palestine exclusivement. L'inter-

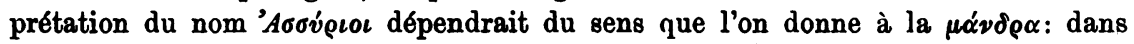
le premier cas, ce terme signifierait les ennemis de Byzance à l'époque de S. André, c'est-à-dire les Arabes et les Bulgares; dans le second, les tyrans arabes qui 
L'interprétation que le P. Vailhé a donnée du texte d'André de Crète n'est donc justifiée en aucune façon. Est-ce à dire que l'opinion de M. de Boor soit définitivement prouvée et qu'il ne se rencontre pas un seul auteur grec «qui ait appelé autrement que Sarrasins, Arabes, Agaréniens les disciples de Mahomet qui attaquaient les Byzantins; avant comme après la translation du califat à Bagdad»? ${ }^{1}$ ) $\mathrm{Ce}$ serait une profonde erreur. Si le P. Vailhé n'a pas eu la main heureuse dans le choix de ses arguments, il n'en est pas moins certain qu'il faut se rallier à son avis, lorsqu'il affirme que M. de Boor s'est trompé et que les Byzantins ont parfois donné le nom d'Assyriens aux sectateurs de l'Islam. La preuve s'en troure dans un texte postérieur de deux siècles au second Anastase, le sixième et dernier livre de la Continuation de Théophane ${ }^{2}$ ), ou on lit, au chapitre concernant

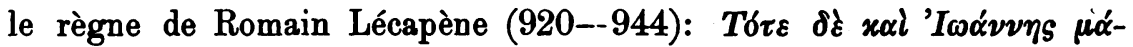

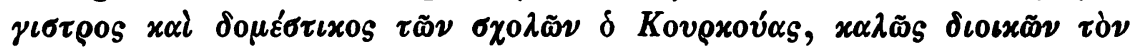

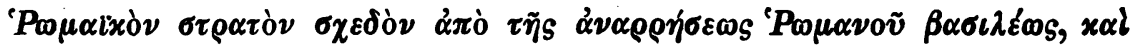

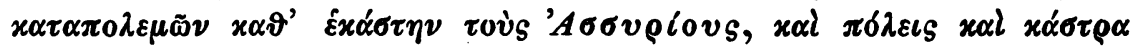

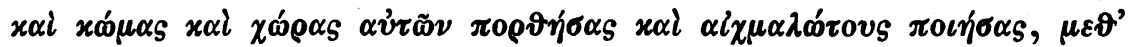

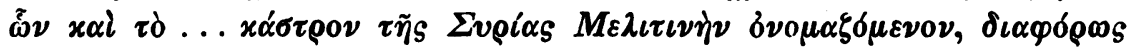

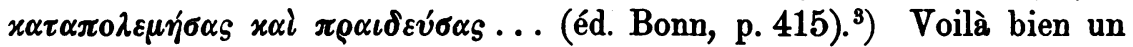
passage désignant les Arabes, d'une manière absolument claire, sous le nom d'Assyriens. Peut-être ne serait-il pas difficile de découvrir d'autres témoignages du même genre; on saurait en même temps si la dénomination d'Assyriens a été donnée aux Arabes sous les califes ommiades de Damas. Pour le moment, nous croyons inutile d'entreprendre cette recherche, parce que toute une série d'exemples, quelque

persécutaient les chrétiens de Palestine au VII siècle. M. P.-K. préfere la deuxième interprétation, parce qu'il ne doute pas que le discours de S. André ait été prononcé à Jérusalem, avant son élévation à l'épiscopat, donc à la fin du VII ${ }^{\circ}$ siècle. A notre avis, rien dans le discours d'André ne justifie cette assertion; lorsque le panégyriste nomme Jérusalem, il le fait à la manière dont on cite une ville étrangère. Ensuite, la prière qu'il adresse pour l'affermissement de l'empire

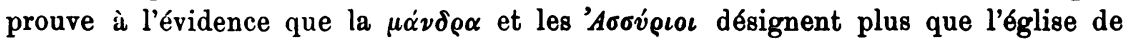
Palestine et ses persécuteurs. Il est très probable, du reste, que les œuvres oratoires d'André de Crète n'ont été composées qu'après son départ de la ville sainte, qu'il quitta tout jeune, lorsqu'il n'était pas encore diacre; cf. Vailhé, Saint Andvé de Crète, Échos d'Orient, t. V (1902), p. 380 et ss.; Ehrhard, dans Krumbacher, Gesch. der byz. Litt. ${ }^{2}$, p. 165.

1) de Boor, ast. cité, p. 635; Vailhé, art. cité, p. 209.

2) Cf. Krumbacher, Geschichte der byzant. Litteratur ${ }^{2}$, p. 348.

3) Au sujet du rôle important joué par Kurkuas dans la guerre contre les Arabes, voir, entre autres, A. Rambaud, L'empire grec au dixième siècle, p. 420 et passim. 
probants qu'ils soient, ne démontrerait pas qu'il faut voir les Arabes dans les Assyriens mentionnés par Romanos dans son cantique sur les dix vierges. Cette série serait contrebalancée, en effet, par l'ensemble

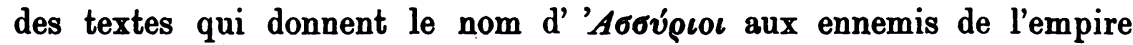
grec qui précédèrent les califes arabes sur le sol asiatique, nous voulons dire les Perses. M. de Boor n'a cité aucun de ces textes, ce qui a fait croire au $P$. Vailhé qu'il n'en existait pas. Mais déjà Procope, dans son traité de la guerre des Perses, emploie assez fréquemment le

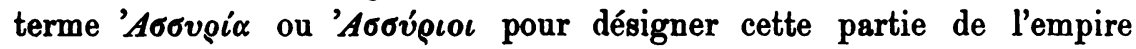
perse qui correspondait à peu près à l'ancienne Assyrie; il y englobe, semble-t-il, la Babylonie, car il indique Séleucie et Ctésiphon comme villes principales. Voici comment il s'exprime, en décrivant le cours

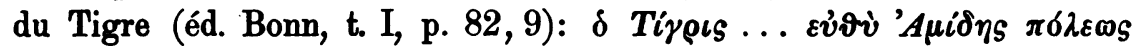

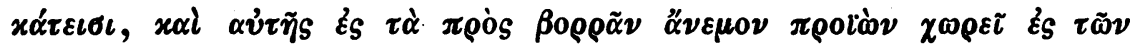
'Acsupíiv $\tau \eta \dot{\nu} \chi \chi^{\omega} \rho \alpha \nu$. La même appellation se rencontre un peu plus loin (p. 85,5), au sujet de l'Euphrate, ainsi que dans les passages

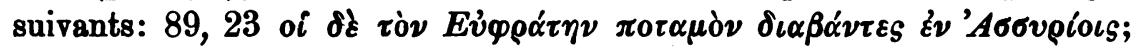

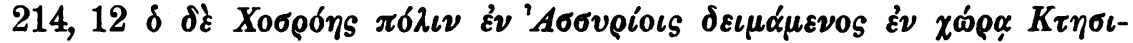

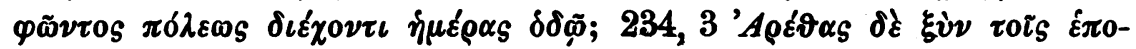

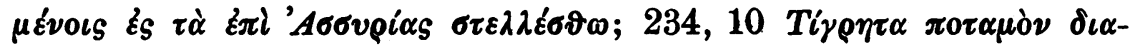

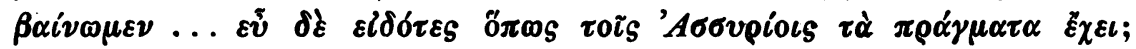

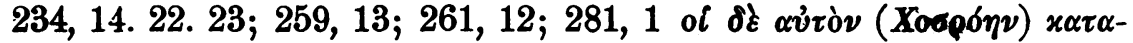

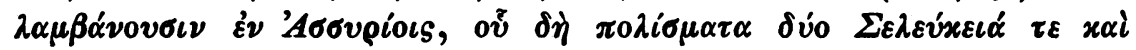

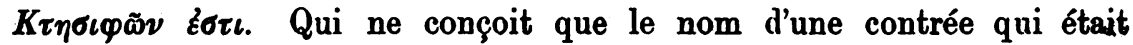
devenue le centre politique de l'empire perse ait pu être donné parfois aux Perses eux-mêmes, dont les conquêtes, sous certains princes Sassanides, rappelaient d'une manière si frappante l'extension de l'ancienne monarchie assyrienne. Théophane (Chronographie, éd. de Boor, t. I, p. 37,11) fait mention des Assyriens vaincus par l'empereur Constance, dans des termes qui ne permettent pas de douter qu'il désigne ainsi les Perses:

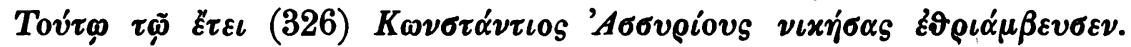

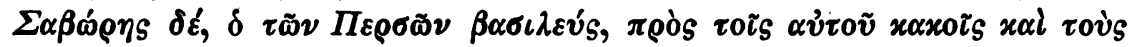

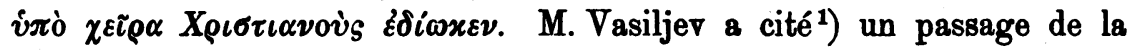
chronique syriaque de Zacharie de Mytilène, qui qualifie d'Assyrien un roi de Perse contemporain de l'empereur Anastase I. ${ }^{2}$ ) D'après l'historien Ménandre (éd. Bonn, p. 360, 22), l'une des clauses du traité de paix conclu en 562 entre les Perses et Byzance concernait les négociants sarrasins qui introduiraient en contrebande dans l'empire grec ou dans

1) Art. cité, p. 464.

2) The Syriac chronicle known as that of Zachariah of Mitylene, translated by Hamilton and Brooks, p. 164. 
l'empire perse des marchandises provenant de l'un de ces deux pays:

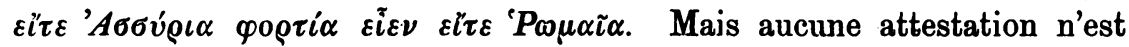
plus formelle que celle d'Arcadius, archevêque de Constantia dans l'île de Chypre (premier tiers du VII ${ }^{e}$ siècle), auteur d'une biographie de $\mathrm{S}$. Syméon stylite le jeune ( $\dagger$ vers 596 ) dont il avait été le disciple. Cette œuvre, sur l'authenticité de laquelle ne peut planer aucun soupçon $^{1}$ ), est malheureusement inédite; on y trouve, exposé d'une façon assez détaillée, le récit de l'expédition du roi de Perse Chosroès en Syrie (540) et de la prise d'Antioche par l'armée de ce prince. Voici quelques extraits de la relation d'Arcadius, d'après le manuscrit d'Oxford, Baroccianus 240 , du XI' siècle, fol. $193^{\vee}$, dont les divergences avec le Monacensis gr. 366, le Parisimus gr. 1459 et le Sabaiticus $108^{2}$ )

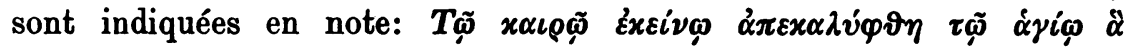

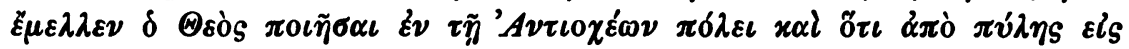

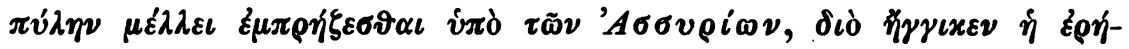

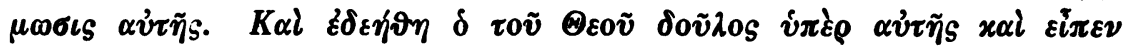

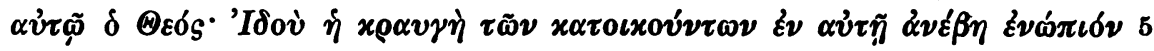

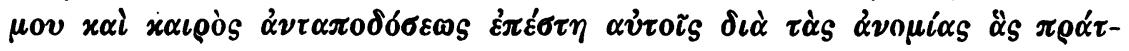

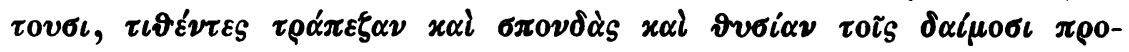

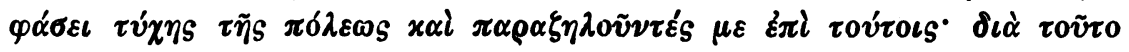

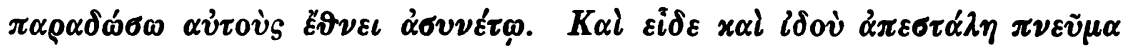

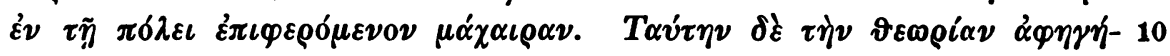

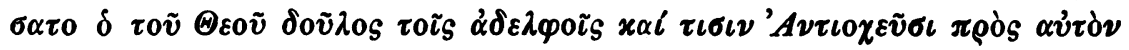

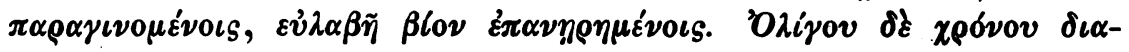

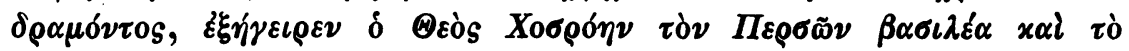

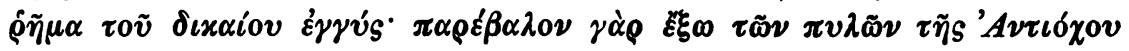

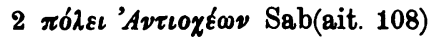

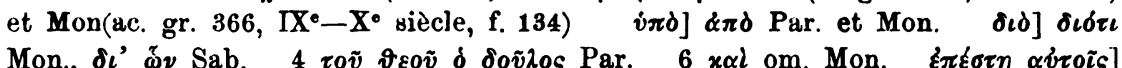

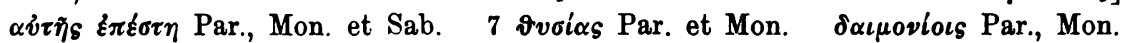

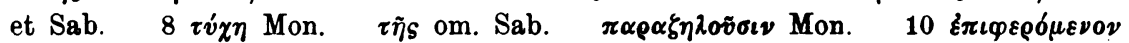
$\mu \alpha^{\prime} \chi \alpha \iota \rho \alpha \nu \varepsilon \nu \tau \tilde{\eta} \pi \delta \lambda_{\varepsilon \iota}$ Par., Mon. et Sab. $\left.11 \tau \iota \sigma \iota v\right] \tau o \tau_{S}$ Sab. $12 \pi \alpha \rho \alpha \gamma \varepsilon v 0-$

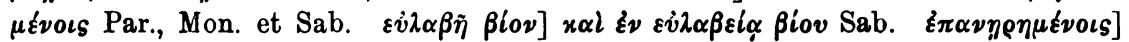

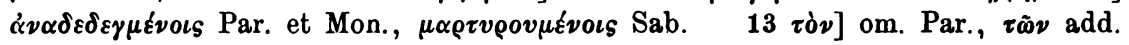
Mon. et Sab. $14 \pi \alpha \rho \varepsilon v \varepsilon \beta \alpha \lambda_{0 \nu}$ Par., Mon. et Sab.

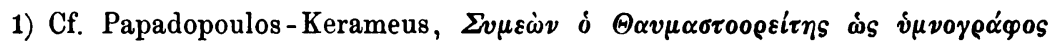

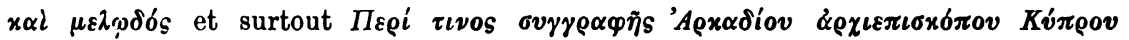

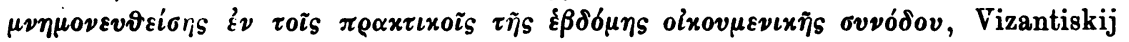
Vremennik, t. I (1894), pp. 141-150, 601-612.

2) Le texte du manuscrit de Saint-Sabas a été reproduit par M. PapaảopoulosKerameus dans l'article de la $N \varepsilon^{\prime} \alpha$ ' $H \mu \varepsilon^{\prime} \rho \alpha$ cité ci-dessus, ce qui nous permet d'en relever ici les variantes. 


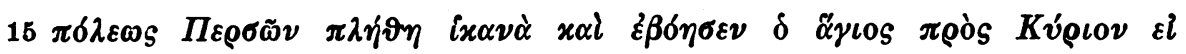

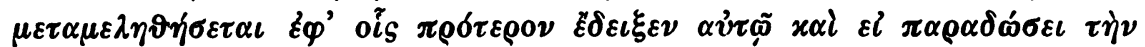

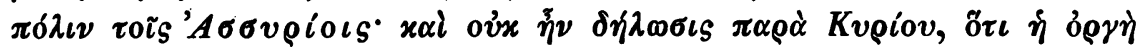

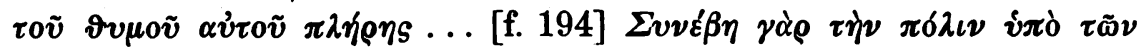

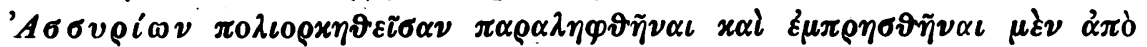

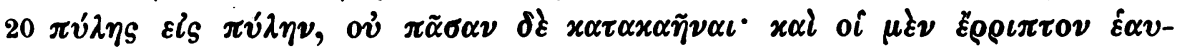

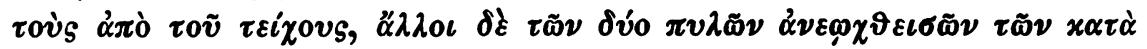

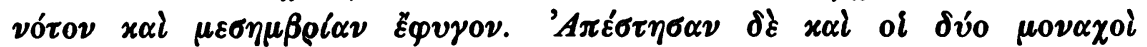

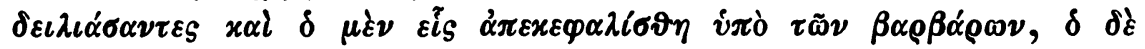

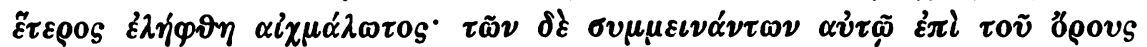

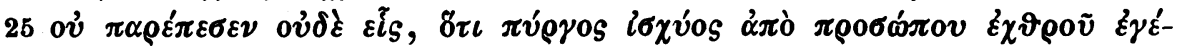

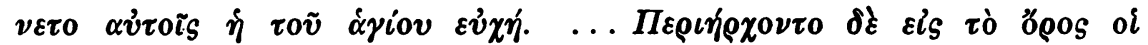

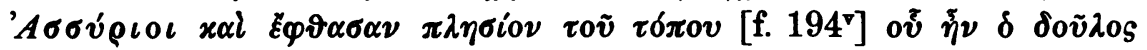

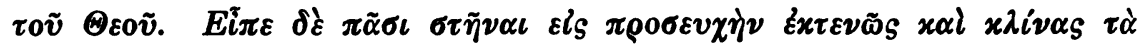

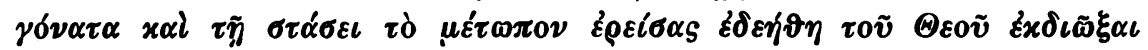

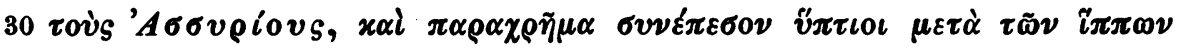

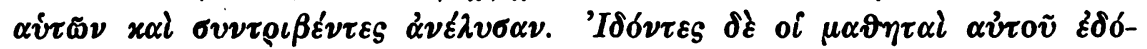

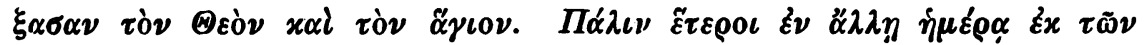

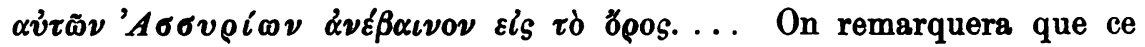
texte si explicite décrit précisément l'invasion persane à laquelle, d'après M. de Boor ${ }^{1}$ ), Romanos ferait peut-être allusion dans son cantique sur les dix vierges.

Que conclure des considérations qui précèdent, sinon qu'on a accordé jusqu'ici une importance beaucoup trop grande, pour établir la chronologie du célèbre mélode, au cantique qui mentionne les Is-

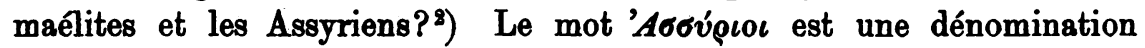
qui n'a de sens relativement précis que dans l'histoire ancienne, tandis que le moyen âge s'est plu à l'appliquer à des peuples et même à des races différentes. Ce n'est pas ici le lieu de rechercher l'origine et le pourquoi de ce singulier fait. Qu'il nous suffise de noter que ce terme d'Assyriens ne se rencontre que rarement dans les textes byzantins en

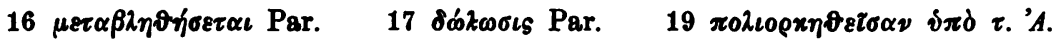

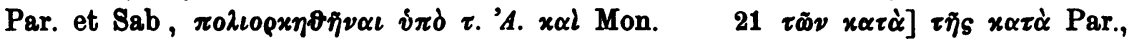

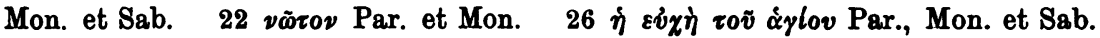

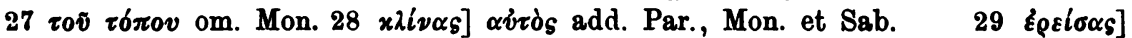

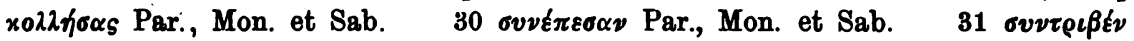



1) Art. cité, p. 639; cf. Papadopoulos-Kerameus, $N \varepsilon \alpha{ }^{~} H \mu \varepsilon \rho \alpha, 29$ juillet 1902.

2) M. Papadopoulos-Kerameus, qui ne connaît que le passage de la Vie de $\mathrm{S}$. Syméon stylite le jeune cité ci-dessus, estime au contraire que l'argument tiré de ce cantique en faveur du VI• siècle garde toute sa valeur. 
dehors des citations bibliques, que son emploi paraît restreint à des écrivains déterminés et enfin, qu'un cas tout analogue nous est fourni par l'histoire du nom de Huns, que Théophane applique également aux Scythes, aux Massagètes, aux Bulgares, aux Turcs et aux Avares. ${ }^{1}$ ) Peut-être faut-il considérer le terme que nous étudions comme un nom géographique servant à désigner, au sens large, les occupants de l'ancien royaume d'Assyrie, c'est-à-dire les Perses et plus tard les Arabes. ${ }^{2}$ ) On peut aussi se demander si le rôle joué par les Assyriens dans la Sainte Écriture n'a pas eu quelque influence sur l'emploi de ce terme dans la littérature byzantine, avec la signification que nous venons d'indiquer.

Bien que Romanos ait pu, par son allusion aux Assyriens, désigner les Arabes aussi bien que les Perses et que la solution du problème chronologique ne dépende en aucune façon de cette phrase tant discutée du cantique sur les dix vierges, il n'en est pas moins certain que l'explication de $\mathbf{M}$. de Boor demeure la plus vraisemblable, parce que seule elle fait comprendre pourquoi le poète oppose les Assyriens aux Ismaélites. L'interprétation du P. Vailhé rencontre, en effet, la même objection que celle de $M$. (Gelzer $\left.{ }^{3}\right)$ : les califes arabes du VIII siècle, qu'on les fasse régner à Bagdad ou à Damas, sont des Ismaélites au même titre que les tribus sarrasines qui dévastaient dans le même temps la Palestine et la Syrie, et on cherche en vain le motif pour lequel le mélode aurait réservé cette dénomination à celles-ci plutôt qu'à ceux-là. Dans tous les textes postérieurs au VII ${ }^{\ominus}$ siècle où nous avons rencontré le nom d'Ismaélites, plus fréquemment employé à cette époque tardive que ne le croit $M$. de Boor ${ }^{4}$ ), ce nom désigne les Arabes comme tels et est tout simplement synonyme

1) Chronographie, éd. de Boor, t. I, aux passages indiqués a l'index. L'éditeur fait remarquer que ce nom a été donné par la plupart des Grecs du moyen âge à tous les peuples septentrionaux.

2) Parfois aussi on rencontre les Assyriens mentionnés à côté des Perses ou des Arabes: cf. Théophane, éd. de Boor, t. I, p. 33, 15; la continuation de Théophane, éd. Bonn, p. 55, 6; pseudo-Syméon magister, éd. Bonn, p. 678, 12 . Il semble que ce terme désigne alors, non plus les conquérants du vieil empire assyrien, mais exclusivement les habitants de l'Assyrie proprement dite, devenue simple province de l'empire perse ou arabe. Le nom $B \alpha \beta v \lambda$ ćvı

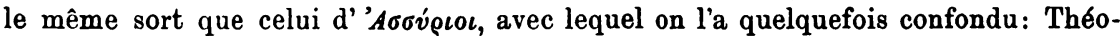
phane (p. 251, 5) reproduisant Théophylacte Simocatte (éd. Bonn, p. 145, 9) l'applique aux Perses contemporains de l'empereur Tibère (578), tandis qu'il appelle $B \alpha \beta v \lambda \omega v i \alpha$ (p. 256, 11) un des territoires en leur possession, probablement l'ancieune Babylonie.

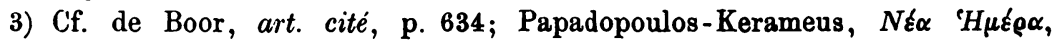
2 juillet $1902 . \quad$ 4) Art. cité, p. 634.

Byzant. Zeitschrift XII 1 u. 2 


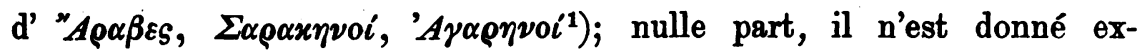
- clusivement à une fraction quelconque des descendants d'Ismaël. Et puis, ne serait-il pas bien étrange qu'énumérant les calamités subies par Byzance, Romanos ait placé sur la même ligne deux facteurs d'importance aussi inégale que les califes arabes et les tribus nomades de Palestine et de Syrie? Les lieutenants du Prophète sont les ennemis les plus redoutables de l'empire grec; ils tiennent en échec ses armées, lui ravissent ses plus belles provinces et ne craignent pas de s'avancer jusqu'aux portes de la capitale. Il n'est donc pas étonnant qu'ils aient une des premières places dans les préoccupations du souverain et du peuple byzantins et que leur nom apparaisse presque à chaque page des chroniques de l'époque. Tout autre est la situation des tribus sarrasines. $\mathrm{Au} \mathrm{VI}^{e}$ siècle, il est vrai, elles constituent une force spéciale dont les Perses comme les Byzantins ont à tenir compte et qui inflige plus diune défaite aux généraux envoyés contre elle par l'empereur grec. ${ }^{8}$ ) Mais au VIII' siècle, complètement englobées dans la monarchie musulmane, elles se contentent de molester les chrétiens et les moines et de s'adonner au brigandage; leur rôle tout. local n'attire guère l'attention des Byzantins, presque entièrement captivée par les incursions que font dans l'empire les Arabes et les Bulgares. La preuve, c'est qu'il n'est question de ces tribus nomades que dans des relations rédigées sur place, là ou s'exerçaient leurs rapines, par des moines qui avaient intérêt à conserver à la postérité le souvenir de leurs frères massacrés par les bandes pillardes ${ }^{3}$ ); jamais on ne les voit

1) Voir la continuation de Théophane, éd. Bonn, p. 11, 2; 97, 5 (le calife de Bagdad est appelé Ismäl); 114, 19; 116, 22; 120, 8 et 16 (Ismaël, pour désigner le calife); 127, 19; 187, 15; 167, 11; 176, 4; 186, 10; 267, 20; 288, 4; 368, 23. Nicétas de Byzance se sert quelquefois du terme «Ismaelites»; cf. Refutatio Mahomedis, Patrol. gr., t. 105, col. 669-806, surtout le chap. 25 intitulé 'A $\pi 6$ -

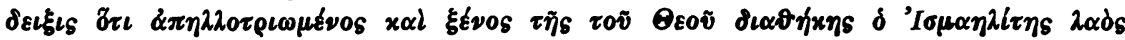

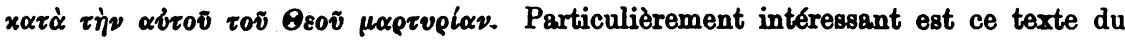

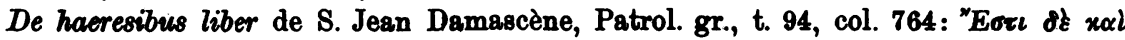

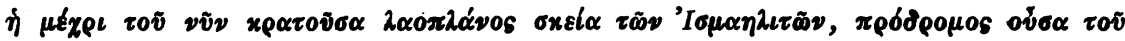

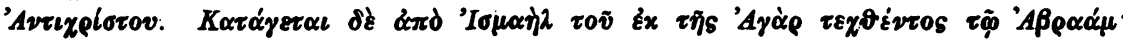

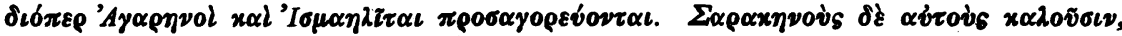

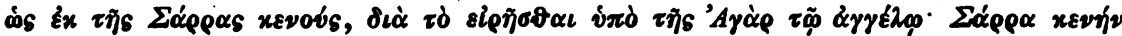

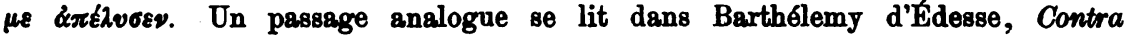
Muthammed, Patrol. gr., t. 104, col. 1448.

2) Cf. de Boor, art. cité, p. 636-639; Vasiljev, art. cité, p. 466-469, où sont signal6́s, entre autres, plusieurs passages intéressants d'écrivains orientanx.

3) Ainsi la Passio sanctorum XX martyrum (fin du VIII' siècle) par Etienne le mélode, moine de Mâr-Saba et témoin oculaire, Acta Sanct., martii t. III, p. 166 et 88. 
mentionnées dans des chroniques générales comme la chronographie de Théophane, qui pourtant signale parfois l'état de la Palestine et de la Syrie sous l'administration des califes ainsi que les vexations et les tourments de toute sorte que subissaient les chrétiens de la part des Arabes de cette région. ${ }^{1}$ ) Du reste, si l'on observe que Romanos cite les Ismaélites en premier lieu: "voici que les Assyriens et avant eux les Ismaélites nous ont amenés prisonniers", on conviendra que l'hypothèse de M. de Boor est la seule qui explique jusqu'ici d'une manière adéquate chacune de ces paroles du poète.

Nous nous arrêterons moins longtemps au second argument positif $^{2}$ ) invoqué par le $\mathrm{P}$. Vailhé en faveur d'Anastase II. Dans le cantique de Romanos sur la présentation de Jésus-Christ au temple, on lit les

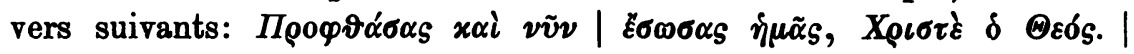

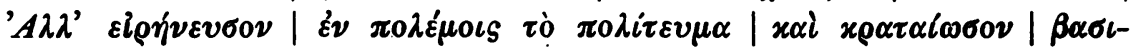
$\lambda \varepsilon^{\prime} \alpha s$, ớs $\eta \gamma_{\alpha}^{\prime} \pi \eta \sigma \alpha .^{3}$ ) Voici la traduction du P. Vailhé: «0 Christ, notre Dieu, toi qui viens à l'instant de nous sauver, ramène la paix dans la ville livrée à la guerre et fortifie les empereurs que tu as aimés». Les deux premiers vers feraient allusion à la délivrance de Constantinople assiégée par les Arabes, le 15 aout 718, le troisième et le quatrième aux guerres civiles provoquées dans la capitale par la révolte d'Anastase II en 719, les deux derniers à l'association au trône de Constantin Copronyme, réalisée par Léon l'Isaurien le 25 mars 720.4) Ce passage établirait donc définitivement la chronologie de Romanos, si, par malheur, l'interprétation proposée n'était fort sujette à caution.

1) Chronographie, éd. de Boor, 452, 22;484, 10;499, 15; etc. Théophane ne mentionne aucun pillage ni aucune persécution contre les chrétiens en Palestine et en Syrie pendant la première moitié du VIII siècle.

2) Le troisième argument du P. Vailhé est purement négatif: il se base sur une étude du même auteur, Saint André de Crète, Échos d'Orient, t. V (1902), p. 380 et ss., d'où il résulte que saint André de Crète est mort le 4 juillet 740, et non pas en 720 , comme on le croyait jusqu'ici. Il n'y aurait donc plus aucune difficulté à admettre que Romanos soit venu à Constantinople sous Anastase II et qu'il y ait composé des cantiques, imités ensuite par saint André.

3) Krumbacher, Studien zu Romanos, p. 185 et s. L'authenticité de cette strophe est mise en doute par l'éditeur, ibid., p. 253 et $\mathrm{s}$.

4) Une interprétation analogue a été proposée, il y a vingt ans déjà, par Jacobi, Zur Geschichte des griechischen Kirchenliedes, Zeitschrift für Kirchen-. geschichte, t. V (1882), p. 204 et ss. Elle est écartée avec raison par M. Vasiljev,

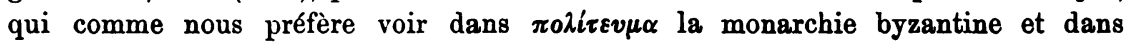
$\beta \alpha \varsigma_{\imath} \varepsilon \tau_{s}$ les ampereurs greç en général (art. citúé, p. 477). M. PapadopoulosKerameus ( $N \xi_{\alpha}{ }^{'} H \mu \varepsilon \dot{\varepsilon} \alpha, 29$ juillet 1902) a prouvé également que l'argument du P. Vailhé repose sur une traduction inexacte du mot $\pi 0 \lambda i \tau \varepsilon v \mu \alpha$. 
Nous ne connaissons aucun dictionnaire traduisant par «ville» le mot

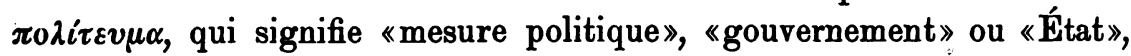
et quand bien même il aurait parfois ce sens, rien ne prouverait que

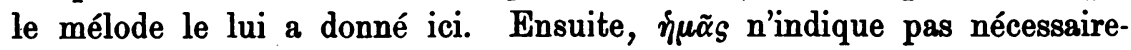
ment les habitants de Constantinople, pas plus qu'il n'a cette signifi-

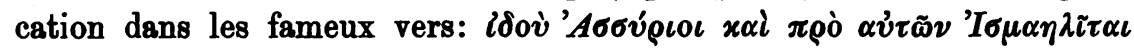

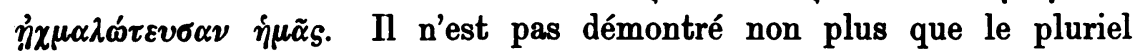
$\beta \alpha \sigma \iota \lambda \varepsilon \tau_{S}$ désigne, surtout dans le langage poétique, deux empereurs associés au trône. ${ }^{1}$ ) Dès lors, le cantique ne fournit plus aucun point de repère, parce que les événements auxquels il fait vaguement allusion se sont présentés au $\mathrm{VI}^{\circ}$ siècle aussi bien qu'au VIII' ou qu'à d'autres périodes troublées de l'histoire de Byzance. On ne peut nier que sous le règne d'Anastase $I$, par exemple, il surgit successivement plusieurs guerres civiles et plusieurs conflits extérieurs, assez graves pour justifier les supplications du poète. Et même, s'il fallait voir dans ces vers une allusion à deux empereurs associés au trône, on ne serait pas embarrassé de découvrir, dans les premières années du $\mathrm{VI}^{\bullet}$ siècle, une circonstance de ce genre. Justin I s'adjoignit, en effet, Justinien comme collègue et le couronna ainsi que son épouse Théodora le $1^{\text {or }}$ avril 527.

Il va sans dire que les observations qui précèdent ne permettent aucunement de dater Romanos du règne du premier Anastase; elles ont pour seul but de montrer que les nouveaux arguments apportés en faveur du VIII siècle ne méritent pas d'être pris en sérieuse considération. Comme l'a dit avec insistance $M$. Krumbacher ${ }^{2}$ ) et après lui $\mathbf{M}$. de Boor $\left.{ }^{3}\right)$, il est impossible pour le moment de déterminer avec certitude l'époque où a vécu le célèbre poète, et cette question si complexe ne pourra être reprise avec quelque chance de succès que lorsqu'on possédera l'édition complète et définitive des œuvres de Romanos que prépare M. Krumbacher. ${ }^{4}$ ) En outre, il faudra étudier

1) C'est la signification qu'il a dans le De cerimoniis de Constantin Porphyrogénète, où son emploi est très fréquent; cf. par exemple pp. 68 et s., 194 et s8., 527, 687 et s8. (éd. Bonn). L'impératrice y est toujours, semble-t-il, mentionnée à

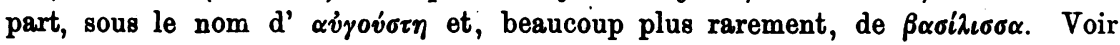
cependant le commentaire de Reiske, qui signale, p. 861,7 , un passage où $\beta \alpha \sigma \imath \lambda \varepsilon i S_{S}$ désigne peut-être le couple impérial.

2) Umarbeitungen bei Romanos, p. $148 . \quad$ 3) Art. cité, p. 640.

4) M. Papadopoulos-Kerameus est plus catégorique. Les arguments qui militent en faveur d'Anastase I lui paraissent absolument décisifs, et les allusions historiques du cantique sur les dix vierges établissent même, d'après lui, avec beaucoup de vraisemblance, que ce cantique a été composé à la fin du règne de 
de près la relation anonyme des miracles de $\mathrm{S}$. Artémius, dans laquelle M. Vasiljevskij a signalé ce passage important: «Un jeune homme chantait des vers du saint philosophe Romanos» ${ }^{1}$ ); le miracle dixhuitième qui contient ce détail, est rapporté par l'hagiographe au règne d'Héraclius $(611-641)$. $M$. Krumbacher a donné d'utiles renseignements bibliographiques sur ce recueil de mirabilia, qui n'est connu jusqu'ici que par une ancienne version slave, et il a conjecturé qu'il était traduit d'un texte grec qui serait à retrouver. ${ }^{2}$ ) Nous sommes à même de compléter ces indications, car le texte grec des miracles de S. Artémius n'est pas à chercher bien loin. Le manuscrit grec 1468 de la bibliothèque nationale de Paris, datant du $\mathrm{XI}^{\circ}$ siècle ${ }^{3}$ ), qui est un recueil de documents hagiographiques pour les mois de septembre, octobre et novembre, offre à la date du 20 octobre,

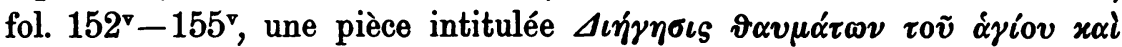

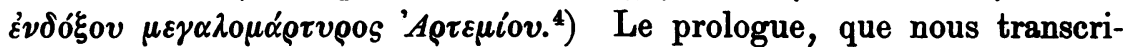
vons en partie, concorde mot pour mot avec le début de la relation russe, dont $M$. Krumbacher a cité les premières phrases en traduction

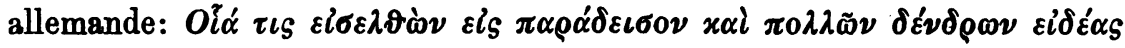

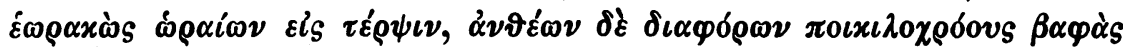

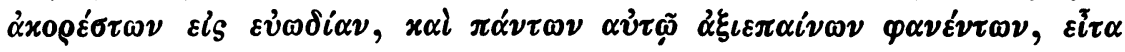

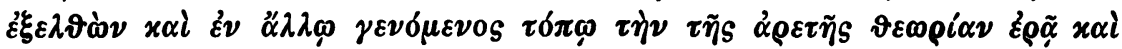

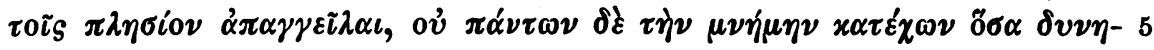

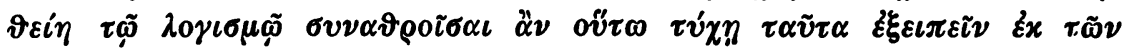

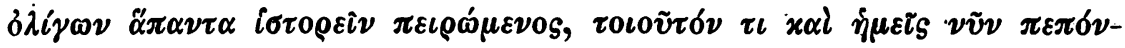

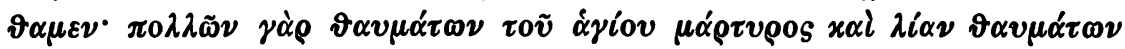

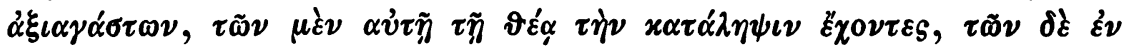

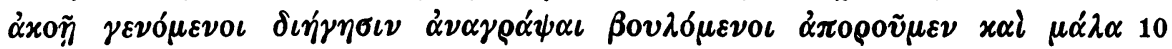



$6 \tau \dot{x} \chi \varepsilon \iota \operatorname{cod}$.

Justinien. Arrivé à Constantinople sous le règne d'Anastase, le poète aurait vécu jusque sous Justin II. Par contre, M. Vasiljev (art. cité, p. 463-475) s'est efforcé de démontrer que le même cantique ne se rapporte qu'à des événements du règne d'Anastase I! De pareilles divergences sont la preuve qu'on ne peut parvenir à aucun résultat précis à l'aide des vagues allusions du cantique de Romanos.

1) Visantiskij Vremennik, t. I (1894), p. 256-258.

2) Umarbeitungen bei Romanos, p. 149 et $\mathrm{s}$.

3) Cf. Catal. codd. hag. graec. bibl. nat. Parisiensis, p. 142 . M. Vasiljev, art. cité, p. 454, signale également ce ms., ainsi que le Coislin 304 (cf. Catal. codd. . . , p. 312). Celui-ci ne renferme qu'un seul miracle (fol. 30-32), qui ne se retrouve ni dans le Paris. 1468 ni dans le Bruxellensis décrit ci-dessous.

4) Nous devons la copie de cette pièce à l'obligeance du R. P. J. van den Gheyn, auquel nous présentons l'expression de notre profonde reconnaissance. 
Le premier chapitre du texte grec comme de la version russe contient l'histoire d'un jeune homme de vingt ans, fils du médecin Anthime, qui dut sa guérison à l'intercession de $\mathrm{S}$. Artémius. Malheureusement, les miracles du manuscrit de Paris ne sont qu'au nombre de six, alors que la recension slave en compte trente-quatre, et quoique le dernier offre le récit d'une guérison merveilleuse opérée sous le règne d'Héraclius, ce n'est pas celui qui renferme le passage concernant Romanos. Ce passage ne se rencontre pas davantage dans la série des miracles de $\mathrm{S}$. Artémius que contient le ms. $8599-600$ de la bibliothèque royale de Bruxelles, formé de plusieurs copies de documents hagiographiques grecs prises au $\mathrm{XVII}^{\circ}$ siècle dans des manuscrits de la bibliothèque de Saint-Sauveur à Messine. Ici les miracles sont au nombre de treize (fol. $179-190^{\top}$ ), précédés du prologue que nous avons cité. D'après un renseignement que nous devons à l'obligeance de M. G. Caracciolo, le manuscrit de Saint-Sauveur dont dérive la copie de Bruxelles porte actuellement le $\mathrm{n}^{\circ} 30$ dans le fonds provenant de ce monastère, qui fait partie de la bibliothèque de l'Université de Messine. Un troisième exemplaire des miracles de $\mathrm{S}$. Artémius est le ms. 27 de Saint-Sabas de Jérusalem, du $\mathrm{XI}^{\bullet}$ siècle, qui ne nous est connu que par la description de M. Papadopoulos-Kerameus. ${ }^{1}$ ) Il comprend dixhuit chapitres, mais il n'est pas possible de dire maintenant si l'un d'eux fait allusion aux poésies de Romanos. Chose à retenir, les trois copies que nous venons de signaler ne different pas seulement par le nombre des chapitres qu'elles contiennent: chacune d'elles a plusieurs miracles en propre, et ceux qu'elles possèdent en commun se présentent dans un ordre qui n'est pas toujours uniforme. Il faudra donc attendre une édition critique et une étude approfondie des miracles grecs de S. Artémins, avant de pouvoir estimer à sa juste valeur l'argument sur lequel s'est appuyé $M$. Vasiljerskij pour dater Romanos du règne d'Anastase I.

Bruxelles.

P. van den Ven.

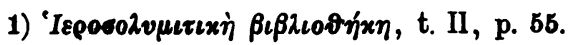

